\title{
Tchad : une mobilisation internationale sans précédent en faveur de l'éducation
}

Emmanuel Bailles

\section{OpenEdition}

Journals

Édition électronique

URL : http://journals.openedition.org/ries/3575

DOI : $10.4000 /$ ries.3575

ISSN : 2261-4265

Éditeur

Centre international d'études pédagogiques

Édition imprimée

Date de publication : 1 décembre 2013

Pagination : 16-18

ISBN : 978-2-85420-601-2

ISSN : 1254-4590

Référence électronique

Emmanuel Bailles, «Tchad : une mobilisation internationale sans précédent en faveur de l'éducation », Revue internationale d'éducation de Sèvres [En ligne], 64 | décembre 2013, mis en ligne le 01 décembre 2015, consulté le 30 avril 2019. URL : http://journals.openedition.org/ries/3575 ; DOI : 10.4000/ ries.3575

Ce document a été généré automatiquement le 30 avril 2019

(c) Tous droits réservés 

sans précédent en faveur de l'éducation

\author{
Emmanuel Bailles
}

1 Le Tchad, pays de 11,5 millions d'habitants à l'espérance de vie moyenne de 49,5 ans, aux trois-quarts rural, est entré dans l'ère pétrolière depuis 2002, multipliant par huit ses ressources budgétaires. Toutefois, le produit intérieur brut par habitant (823\$) et l'indice de développement humain $\left(183^{\mathrm{e}} \text { pays } / 187\right)^{1}$ restent faibles dans ce pays de jonction EstOuest et Nord-Centre de l'Afrique, au cœur de crises régionales (crises sahélienne, libyenne, soudanaise, centrafricaine et nord-nigériane). Le développement du secteur de l'éducation et de la formation vise à sortir le Tchad de son enclavement géographique mais aussi de l'isolement dans lequel il est souvent placé en raison des aléas politiques et militaires que le pays connaît depuis la fin des années soixante.

Dans le contexte du début des discussions internationales sur l'agenda « post-2015 »', les premiers bilans des politiques mises en œuvre par les États d'Afrique subsaharienne sont dressés. L'objectif du millénaire pour le développement du secteur éducation, c'est-à-dire l'atteinte d'une scolarisation primaire universelle (SPU) en 2015, ne sera pas atteint, mais le taux moyen d'achèvement du primaire, principale mesure de la SPU, s'élevait à $70 \%$ environ en 2011, alors qu'il n'était que de $43 \%$ en $1999^{3}$. S'il montre les efforts importants menés par les États en faveur de l'éducation, cet indicateur alerte sur une forte rétention des élèves en cours de cycle, donc d'un taux de redoublement élevé souvent synonyme, in fine, d'abandon de la scolarité et donc d'exclusion du système scolaire.

\title{
Scolarisation primaire universelle et formation des enseignants au Tchad
}

Parmi les exceptions à cette dynamique régionale encouragée par les bailleurs de fonds bilatéraux et multi-latéraux, le Tchad présente un taux d'achèvement du primaire de $35 \%$ 
environ ${ }^{4}$. La faiblesse de ce taux, dans ce pays de $1284000 \mathrm{~km}^{2}$ aux fortes disparités régionales, est à relativiser dans la mesure où seules $48 \%$ des écoles primaires du Tchad ont un cycle complet ${ }^{5}$. Mais les difficultés d'évolution vers la scolarisation universelle ne peuvent s'expliquer par ce seul phénomène. Le système éducatif s'est en effet développé lors de périodes d'accalmie plus ou moins longues, durant lesquelles la volonté de continuité des services de l'État s'est conjuguée aux nécessités de reconstruction politique et sociale dites « post-conflit».

Dans ce contexte de faiblesse de l'offre éducative, les familles tchadiennes se sont saisies de l'organisation des scolarisations primaires en recrutant et en rémunérant elles-mêmes les enseignants de leurs enfants. De cette mobilisation remarquable, il résulte qu'en 2010-2011, $74 \%$ des enseignants du primaire sont "communautaires ", c'est-à-dire recrutés et rémunérés par les familles. Si cette implication forte des familles peut être vue de façon positive, il faut cependant noter que les enseignants communautaires sont trop souvent les enseignants pauvres des familles les plus pauvres, et que la qualité de l'éducation n'est pas garantie en raison de la formation insuffisante des personnes recrutées, choisies parmi les mieux alphabétisées dans un pays où $78 \%$ de la population est analphabète ${ }^{6}$.

5 La Loi d'orientation du système éducatif du 24 février 2006, instruite sur la base d'un diagnostic complet du système éducatif réalisé par les ministères en charge de l'éducation et de la formation, avec l'appui d'une équipe internationale ${ }^{7}$ du Pôle de Dakar de l'Unesco et de la Banque mondiale, porte création d'un corps d'enseignants contractuels et institue la nécessité d'une formation et d'une rémunération minimales, afin de relever la qualité des acquis de l'apprentissage. Cette politique nationale, qui peut sembler se substituer à des initiatives communautaires ayant trouvé un équilibre entre niveau de formation minimal et rémunération décente, vise à garantir une cohérence globale du système éducatif.

6 La formalisation du recrutement des enseignants est aussi un enjeu de justice sociale et donc d'équilibre sociétal: les dernières mesures effectuées par le Programme d'analyse des systèmes éducatifs (Pasec) de la Confemen ${ }^{8}$ indiquent qu'entre 2004 et 2010, le niveau d'acquisition scolaire des élèves a baissé en deuxième année du primaire tout en augmentant en cinquième année, ce qui laisse penser que seuls les élèves issus des familles les plus favorisées demeurent scolarisés'.

\section{Mobilisation nationale et internationale}

7 À l'initiative du Président de la République du Tchad ${ }^{10}$, le gouvernement a organisé en septembre 2012 le Forum national de réflexion sur le système éducatif tchadien. Vingt-etune recommandations ont été adoptées, couvrant l'ensemble des thématiques du système éducatif dans son ensemble (faible qualité des acquis de l'apprentissage, besoins en formation des enseignants, développement de la formation professionnelle, généralisation de l'enseignement bilingue ${ }^{11}$, adaptation du temps scolaire au contexte climatique et agricole, etc.).

En amont du Forum national, durant la même année, la Stratégie intérimaire pour l'éducation et l'alphabétisation (SIPEA) a été adoptée pour la période 2013-2015. La SIPEA est un préalable à la mise en place d'un plan décennal de développement de l'éducation et de l'alphabétisation (PDDEA) de 2015 à 2025. L'enjeu est de taille car le Partenariat 
mondial pour l'éducation (PME) ${ }^{12}$ a accordé au Tchad un appui financier d'un montant de 47,2 M\$ en novembre 2012. Les deux agences des Nations-Unies, l'Unesco et l'Unicef, ont été chargées de gérer ce fonds. D'autres bailleurs ont annoncé leur soutien au secteur de l'éducation: le Qatar (13 M\$), la Coopération suisse (2 MFS), la Banque islamique de développement (12,5 $\mathrm{M} \$)$, la Banque mondiale (15 $\mathrm{M} \$)$, l'Agence française de développement $(5 \mathrm{M} €)$ et des opérateurs, dont le Centre international d'études pédagogiques. En 2014, le Tchad élaborera un nouveau rapport d'État sur le système éducatif national (Resen) avec un nouvel appui d'une équipe international.

Ce soutien massif des partenaires du Tchad, à la hauteur des besoins fondamentaux du système éducatif, renforcera les attentes des populations tchadiennes en matière de développement de l'éducation. Le système éducatif n'est pas un isolat: il est à la fois le produit et le moteur d'un pays composé de familles aspirant à la paix.

\section{NOTES}

1. Données Banque mondiale, 2011.

2. L'« agenda post-2015 » définira, à la suite des Objectifs du millénaire pour le développement à l'horizon 2015, votés en assemblée générale des Nations Unies en septembre 2000, de nouveaux objectifs communs à l'ensemble des pays du monde, quel que soit leur niveau de développement. La question de la durabilité économique et écologique des sociétés humaines devrait être au centre de ces nouveaux objectifs.

3. Source : Rapports Éducation pour tous 2011 et 2012, Unesco.

4. Source : Pôle de Dakar de l'Unesco.

5. Ibid.

6. Recensement général de la population et de l'habitat, 2009.

7. Éléments de diagnostic pour une politique éducative nouvelle et une meilleure efficacité de la dépense publique, Rapport d'État du système éducatif national (Resen), 2005.

8. Confemen : Conférence des ministres de l'Éducation des pays ayant le français en partage.

9. Améliorer la qualité de l'éducation au Tchad: quels sont les facteurs de réussite? Confemen et ministère de l'Enseignement primaire et de l'Éducation civique du Tchad, 2012.

10. Idris Deby Itno, Président du Tchad depuis le $1^{\mathrm{er}}$ décembre 1990.

11. Inscrit dans la Constitution du 31 mars révisée, adoptée par référendum le 6 juin 2005, l'enseignement bilingue français/arabe se développe progressivement. L'arabe enseigné est un arabe classique différent de l'arabe tchadien, langue véhiculaire très usitée. En 2010, $9 \%$ des écoles étaient arabophones ou bilingues, scolarisant $10 \%$ des élèves du primaire, et la moitié des élèves des 21 écoles normales d'instituteurs étudiaient en enseignement bilingue.

12. Le PME, ex-Initiative de mise en œuvre accélérée de l'Éducation pour tous, créé en 2002, fournit des aides financières aux plans nationaux pour l'éducation des pays en développement partenaires, via un fonds fiduciaire pluri-donateurs, le Fonds mondial pour l'éducation. 
INDEX

Mots-clés : aide au développement, coopération internationale, système scolaire, scolarisation Index géographique : Tchad

Keywords : development aid, international cooperation, school system, schooling

Palabras claves : ayuda al desarrollo, cooperación internacional, sistema escolar, escolarización

\section{AUTEUR}

\section{EMMANUEL BAILLES}

Chargé de programme au département coopération en éducation du Centre international d'études pédagogiques. Titulaire d'une maîtrise de philosophie (Université Paris IV - Sorbonne) et d'un master de coopération internationale en éducation et formation (Université Paris V René-Descartes), il a travaillé dans plusieurs pays d'Afrique subsaharienne sur les questions éducatives. 